\title{
Maximization of Service Flows Rates as a Solution of Network Capacity Allocation Problem
}

\author{
Bohdan Buhyl ${ }^{1, \mathrm{a}}$, Pavlo Huskov ${ }^{1, \mathrm{~b}}$, Orest Lavriv ${ }^{1, \mathrm{c}, *}$, Roman Bak ${ }^{1, \mathrm{~d}}$ and Andriy Luntovskyy ${ }^{2, \mathrm{e}}$ \\ ${ }^{1}$ Department of Telecommunications, Lviv Polytechnic National University, Lviv, Ukraine \\ ${ }^{2}$ BA Dresden Univ. of Coop. Education, Dresden, Germany \\ a.bohdan.a.buhyl@lpnu.ua,b.pavlo.o.huskov@lpnu.ua,c.orest.a.lavriv@lpnu.ua, \\ d.roman.i.bak@lpnu.ua,e.andriy.luntovskyy@ba-dresden.de \\ *corresponding author
}

Keywords: Network resources allocation, Service flow, Information services, Serviceoriented resource planning, Network routing.

Abstract: The problem of network capacity allocation is a well-known problem in information and telecommunication area. Emerging set of new services such as cloud-based services, internet of things services, health care services, delivery support services are the key motivators of network capacity growth. In general, network capacity allocation is the static solution for general resources balancing dynamic problem. As far as user demands to change rapidly the network should be capable to support all traffic in accordance with service quality indicators. The solution of the formulated problem is the set of maximum rates of the corresponding service flows that compete with other service flows. This information is essential for the implementation of the service-oriented resource planning, as it enables to calculate the customers' audience if the values of services popularity and subscriber's distribution in the access network are predefined.

Original article, Published date: 2018-05-01

DOI: 10.23977/iotea.2017.31001

ISSN: ISSN 2371-8609 (Print), ISSN 2371-8617 (Online)

https://www.clausiuspress.com/journal/IoTEA.html

\section{Introduction}

In the modern telecommunication networks, the data flow analysis for the service quality provision to end users is an important sophisticated task. The flow rate depends on the number of users that are connected to the local network segment, types of services and the data they transmit. Modern telecommunication networks are content-oriented; therefore, it is important for users to get high- 
quality information and telecommunication services. Some amount of resource has to be allocated to the service flows to deliver these services [1]. Consequently, management and allocation of network resources among multiple service flows to assure high-quality service delivery is an urgent scientific challenge [2,3].

The problem considered in the paper, actually, may be formulated as a question: in case of multiple service flows how much throughput can we allocate to each service flow at every point of the network?

The paper is related to the research area, which defines the used throughput fractions when users access the services [4,5]. Some researchers propose methods and techniques of network resources allocation excluding the impact of the routing process and multiservice traffic characteristics $[6,7]$. However, these papers do not consider the impact of the logical structure on the service flows transmission.

It is very important point to provide an abstraction of the problem to assure its independence from any network technologies and/or protocols. They can only be applied at the stage of some case study.

The authors of the current paper previously have proposed the method of service-oriented resource planning [8], the concept of service assurance in heterogeneous service-oriented systems [9] and methodological basis of heterogeneous network capacity distribution among service flows [10]. Based on the mentioned papers we performed a detail numerical analysis of the network resources allocation to the service flows using the representation of the problem as linear programming problem. This approach has a limitation in the use because of the nonlinear and stochastic nature of network processes. Anyway, it can be applied in case of services flow rates are constant values. This situation can only be observed during very short time series. Therefore, the solution of the problem has a temporal effect and should be recalculated each time the service flow rates changes sensitively. The main advantage of the proposed approach is in operations simplicity and consequently - in duration of computational duration. Typically, computation of the nonlinear optimization problem solution is much more time-consuming even though it can be applied for a long-time perspective. Computational complexity is much higher too. In many cases, it is too hard to implement the nonlinear solution in practice. Therefore, the major part of scientific society tries to convert nonlinear problems to linear ones if it is possible. This process requires the simplification of solution limitations to linear functions too.

\section{Methods and Techniques}

The network resource is represented by the overall capacity of the channels that connect network devices. The amount of the resource allocated to the flow depends on the predefined network structure (network topology). The service flow rate depends on the channel throughput and is limited by other flows between the same pair of nodes. The set of all routes forms the network logical structure. This logical structure is the limiting factor as far as physical resources cannot be utilized completely after the logical structure is organized. Thus, the process of logical structure formation is the determining factor in the service flow rates maximization. The problem is common for all types of networks considering wired or wireless. The distinction only comes when we analyse the network specific protocols are using for logical structure formation. In the current case, we will analyse the most common network layer protocols such as RIP v.2 and OSPF. Anyway, the covered problem is not limited to such protocols. It may be extended using wireless resource allocation protocols. In general, the current problem arises when the multiple access to shared resources is performed. Its solution may be achieved by maximizing the resources allocated to a 
specific demander. In our case, the demander is service flow which should be transmitted over the network.

It is known that the formation of the logical structure is either static (which is very rare), or dynamic (using dynamic routing protocols); or a combination of both approaches. Dynamic routing can be applied by different criteria selection of the route: the metric of the lowest number of hops along the route (RIP - Routing Information Protocol), or of the channel conditions (OSPF - Open Shortest Path First). Logical structures formed by each of the protocols are usually not the same, and therefore the fraction of the allocated physical resources may be different because of different network load, therefore the routing protocol directly impacts on the desired rate of each service flow.

By the flow we mean the traffic of information packets between a pair of nodes, which is transmitted by a single route.

Identifying the flow with the route, the problem of network resources allocation can be formulated in terms of linear programming problem as follows: maximize the rate of each service flow at the input of some network node providing simultaneous existence and equitable competition of the flows and estimate the maximum number of clients using the service with multiple levels of service quality.

A telecommunication network is represented by the graph $G=(V, E)$, with logical structure

$$
L=\{\mu(1,2) ; \mu(1,3) ; \ldots ; \mu(i, j)\}
$$

where $i, j \in V$ formed by the dynamic routing algorithm. The logical structure restricts the use of physical resources, presented by a vector $\vec{x}$. The calculation of the elements of this vector may be performed by solving a linear programming problem, presented by a following system of inequalities:

$$
\begin{aligned}
A \times x & \leq b \\
\min _{x} f^{T} x, \quad A e q \times x & =b e q \\
e b \leq x & \leq u b
\end{aligned}
$$

where $f(x)$ - the objective function; $A, A_{e q}$ - coefficients of linear equations; $x$ - the desired variable; $e b, u b$ - upper and lower bounds of the desired variable.

Let us write the optimization problem in the following form:

$$
\min _{x} f(x)
$$

where $f(x)$ - linear objective function, defined as

$$
f(x)=-\sum_{(i, j)} x_{i, j}
$$

where $i, j$ - number of nodes, $x_{i, j}$ - a variable that defines the rate of the service flow between a pair of nodes. The solution of the problem should satisfy the system of limitation conditions: 
- limitation on a node’s performance:

$$
\sum_{j \in \mu(i, j)} x_{i, j}+2 \cdot \sum_{k \in \mu(i, j)} x_{k, j}+\sum_{i \in \mu(i, j)} x_{i, j} \leq \sum_{j \in V} C_{i, j}
$$

where $C_{i, j}$ - the capacity of the channels $(i, j)$ (in arbitrary units (a.u.)), $\mu(i, j)$ - the route between the pair of nodes $i, j$;

- limitation on channel throughput:

$$
\sum_{i, j \in \mu(i, j)} x_{i, j} \leq C_{i, j}
$$

- limitation on competition of information flows:

$$
0,5 \cdot \frac{C_{i, j}}{N_{\mu(i . j)}^{\max }} \leq x_{i, j} \leq C_{i, j}
$$

where $N_{\mu(i . j)}^{\max }$ - the maximum number of flows transmitting in the channel $(i, j)$, which belongs to the route $\mu(i, j)$.

These conditions were imposed to assure the equal competition among network flows through the features of linear programming methods. We have concluded if two or more flows are transmitting in the network channel, the flow on shortest path gets all the resources, forcing down other flows.

A solution to the formulated problem may be achieved using MatLab system; it was chosen because it includes functions for linear optimization and enables operations with graphs. For this purpose, we used two libraries - Bioinformatics Toolbox [11] and Optimization Toolbox [12]. Bioinformatics Toolbox contains methods for creating, analysing, processing and visualization of graphs. Optimization Toolbox implements numerous algorithms of linear and nonlinear optimization. Using these methods, we developed the software model of the telecommunication network and solved the problem of network resource allocation among non-prioritized service flows providing their rates maximization.

Using optimization function biograph() a graph is represented as a graph object. Using the function shortestpath() we were able the calculate the shortest paths between each pair of nodes in the presented graph. The resulting routes together with weights of graph edges are the input data for the optimization task. Since the problem to be solved belongs to a class of linear programming, we use the function $\operatorname{linprog}()$ based on the algorithm Interior point [12].

Solving the problem of capacity allocation should be performed for the specific implementation of the telecommunication network. In our case study, it is represented by a weighted graph with 9 vertexes and 16 edges (Figure 1). Based on this graph we have calculated the routes between each pair of nodes for the two cases: the usage of RIP routing protocol and the usage of OSPF routing protocol. We suppose that the network uses only single-path routing. 


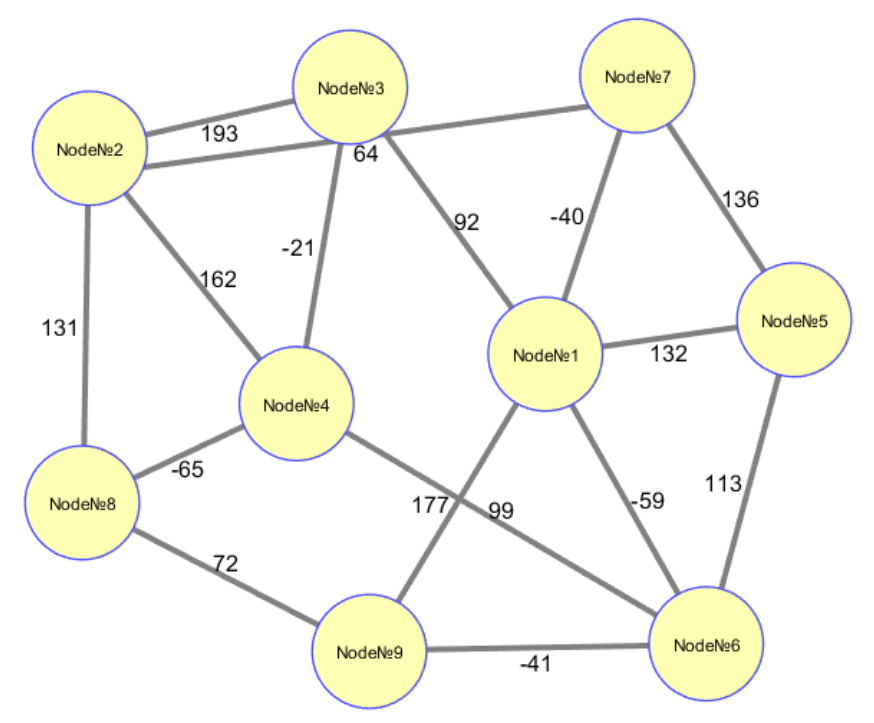

Figure 1: The graph of the studied telecommunication network.

\section{Results and Discussion}

According to equations (4)-(7) for a given graph, the objective function and linear restrictions are formed. Based on them we solved the problem of the network capacity allocation among service flows with the set their rates that is the subject to maximization. These rates are represented by the vector. The optimization problem solutions related to the interior point method are placed in tables 1 and 2.

Table 1. The solution of service flow rates maximization problem in case of OSPF routing.

\begin{tabular}{|c|c|c|c|c|}
\hline Residuals & $\begin{array}{c}\text { Primal Infeas } \\
A \cdot x-b\end{array}$ & $\begin{array}{c}\text { Dual Infeas } \\
A^{\prime} \cdot y+z-f\end{array}$ & $\begin{array}{c}\text { Duality Gap } \\
x^{\prime} \cdot z\end{array}$ & Total Rel Error \\
\hline Iteration 0 & $9.86 \cdot 10^{3}$ & 52.2 & $5.91 \cdot 10^{4}$ & $7.20 \cdot 10^{3}$ \\
\hline Iteration 1 & $9.69 \cdot 10^{2}$ & 2.62 & $7.60 \cdot 10^{3}$ & $8.93 \cdot 10^{-1}$ \\
\hline Iteration 2 & 90.00 & $1.03 \cdot 10^{-1}$ & $1.38 \cdot 10^{3}$ & $5.35 \cdot 10^{-1}$ \\
\hline Iteration 3 & 8.94 & $7.51 \cdot 10^{-3}$ & $1.78 \cdot 10^{2}$ & $1.61 \cdot 10^{-1}$ \\
\hline Iteration 4 & $6.90 \cdot 10^{-1}$ & $1.90 \cdot 10^{-3}$ & 44.30 & $5.25 \cdot 10^{-2}$ \\
\hline Iteration 5 & $2.56 \cdot 10^{-1}$ & $3.32 \cdot 10^{-4}$ & 16.80 & $2.01 \cdot 10^{-2}$ \\
\hline Iteration 6 & $5.26 \cdot 10^{-2}$ & $2.62 \cdot 10^{-13}$ & 3.66 & $4.36 \cdot 10^{-3}$ \\
\hline Iteration 7 & $2.98 \cdot 10^{-4}$ & $1.52 \cdot 10^{-13}$ & $3.15 \cdot 10^{-2}$ & $3.87 \cdot 10^{-5}$ \\
\hline Iteration 8 & $1.50 \cdot 10^{-8}$ & $2.25 \cdot 10^{-14}$ & $1.59 \cdot 10^{-6}$ & $1.96 \cdot 10^{-9}$ \\
\hline Iteration 9 & $2.19 \cdot 10^{-12}$ & $1.64 \cdot 10^{-14}$ & $1.59 \cdot 10^{-14}$ & $2.01 \cdot 10^{-15}$ \\
\hline Iteration 10 & $1.27 \cdot 10^{-13}$ & $9.42 \cdot 10^{-16}$ & $3.98 \cdot 10^{-17}$ & $1.17 \cdot 10^{-16}$ \\
\hline
\end{tabular}

The optimal solution of presented linear programming problem in case of OSPF routing is achieved at 10 iterations. 
Table 2. The solution of service flow rates maximization problem in case of RIP routing.

\begin{tabular}{|c|c|c|c|c|}
\hline Residuals & $\begin{array}{c}\text { Primal Infeas } \\
A \cdot x-b\end{array}$ & $\begin{array}{c}\text { Dual Infeas } \\
A^{\prime} \cdot y+z-f\end{array}$ & $\begin{array}{c}\text { Duality Gap } \\
x^{\prime} \cdot z\end{array}$ & Total Rel Error \\
\hline Iteration 0 & $7.90 \cdot 10^{3}$ & 52.80 & $6.09 \cdot 10^{4}$ & $7.20 \cdot 10^{3}$ \\
\hline Iteration 1 & $1.30 \cdot 10^{3}$ & $1.18 \cdot 10^{-14}$ & $1.24 \cdot 10^{4}$ & 1.17 \\
\hline Iteration 2 & $1.43 \cdot 10^{2}$ & $5.61 \cdot 10^{-14}$ & $2.55 \cdot 10^{3}$ & $5.84 \cdot 10^{-1}$ \\
\hline Iteration 3 & 26.60 & $3.30 \cdot 10^{-14}$ & $4.19 \cdot 10^{2}$ & $2.04 \cdot 10^{-1}$ \\
\hline Iteration 4 & 1.61 & $6.64 \cdot 10^{-14}$ & 37.70 & $5.93 \cdot 10^{-3}$ \\
\hline Iteration 5 & $3.49 \cdot 10^{-1}$ & $1.15 \cdot 10^{-13}$ & 8.93 & $3.62 \cdot 10^{-5}$ \\
\hline Iteration 6 & $2.77 \cdot 10^{-4}$ & $1.17 \cdot 10^{-14}$ & $4.39 \cdot 10^{-2}$ & $1.86 \cdot 10^{-9}$ \\
\hline Iteration 7 & $1.51 \cdot 10^{-8}$ & $1.21 \cdot 10^{-14}$ & $2.26 \cdot 10^{-6}$ & $1.31 \cdot 10^{-15}$ \\
\hline Iteration 8 & $3.71 \cdot 10^{-13}$ & $1.11 \cdot 10^{-14}$ & $1.25 \cdot 10^{-13}$ & $3.79 \cdot 10^{-16}$ \\
\hline Iteration 9 & $1.18 \cdot 10^{-13}$ & $3.21 \cdot 10^{-15}$ & $3.13 \cdot 10^{-16}$ & \\
\hline
\end{tabular}

The optimal solution of presented linear programming problem in case of RIP routing is achieved at 9 iterations.

The solution for RIP protocol is depicted in Figure 2 (bidirectional flows, blue corresponds to the flow rates from source vertex to destination, yellow - vise versa) and for OSPF protocol at Figure 3.

Presented results depict the maximal flow rates that may be achieved in the network presented by a graph at Figure 1 in case of using either RIP or OSPF protocol. We observe that rates are different in each case. It means the studied protocols form different restrictions on network resources utilization as was mentioned above. The numerical results give us better understanding of the traffic processes in the given network.
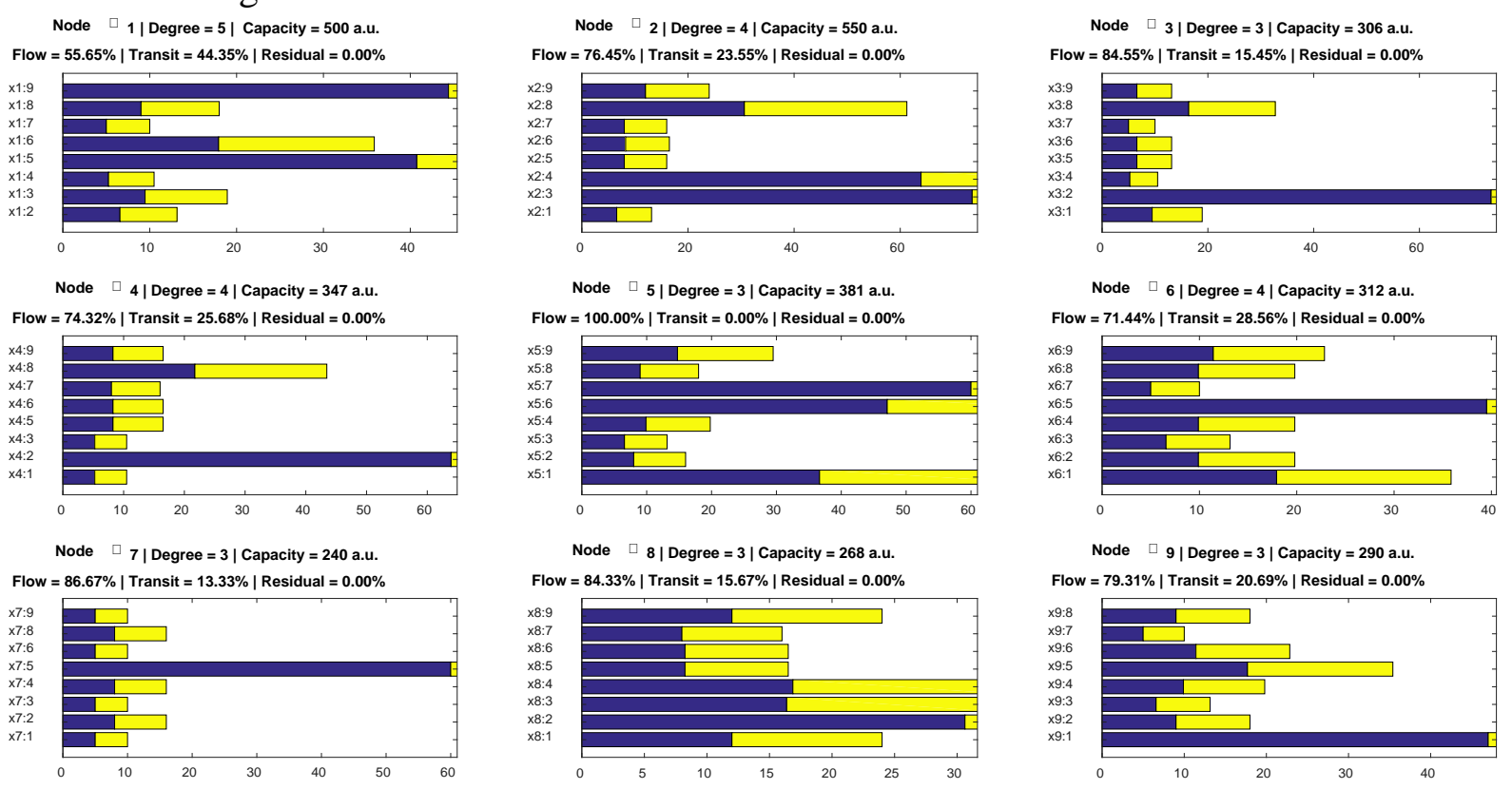

Figure 2: Service flows rate between each pair of nodes in a logical structure formed by the RIP protocol. 

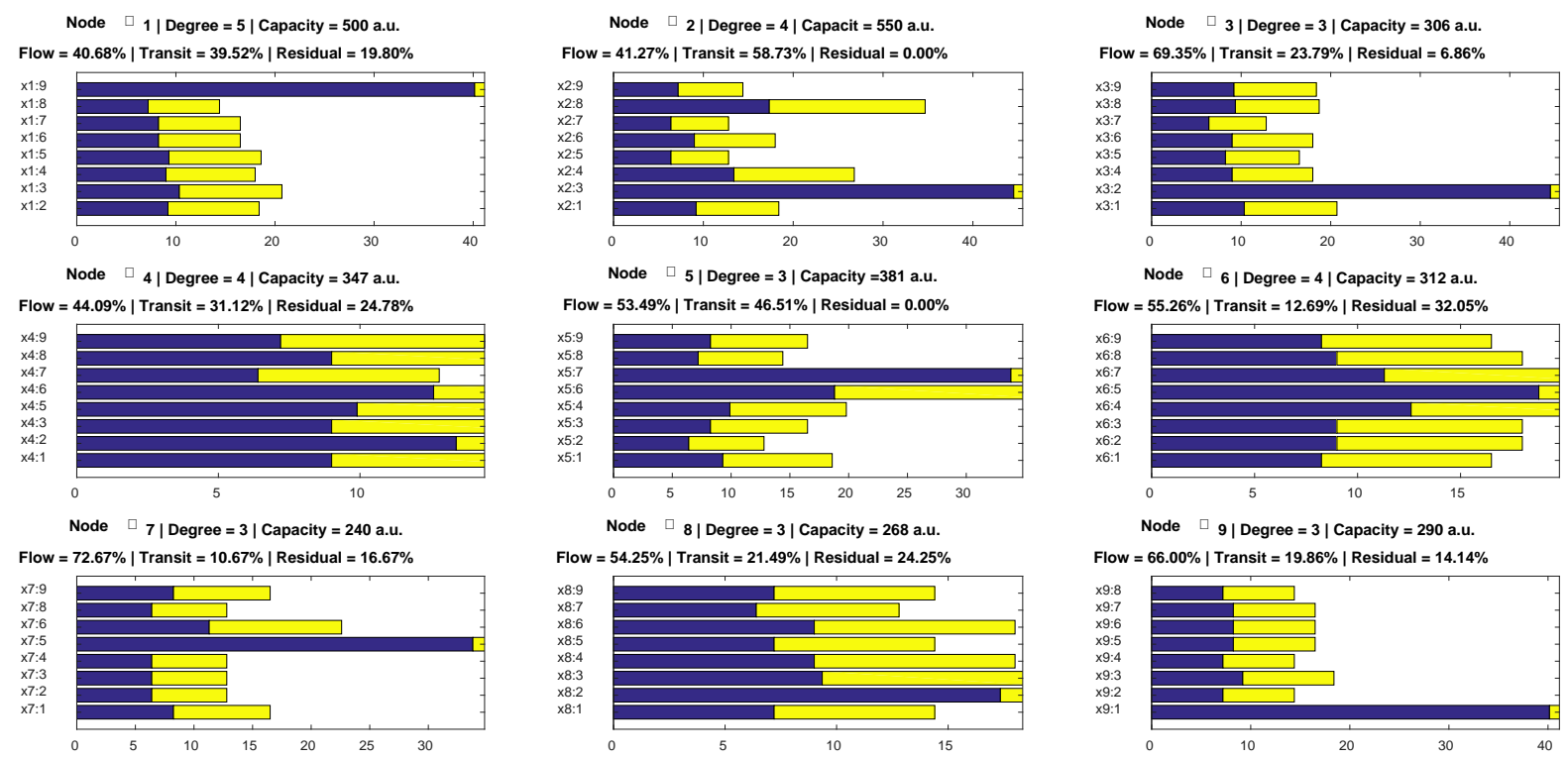

Figure 3: Service flows rate between each pair of nodes in a logical structure formed by the OSPF protocol.

The purpose of knowing the maximal rates of service flow transmitted in the specific network is to be able to manage services in upper network model layer to correspond the user demands. For example, if the total user demand is much higher than the achievable maximal service flow rate, we may either apply the prioritization strategy to give the service to users with high priority or move down the service quality to be sure we are able to serve the required number of the network sessions.

Figure 4 depicts a comparative analysis of the results. The logical further action is to determine the reasons of mentioned distinctions. As far as we know about network routing it may be caused by the metrics both RIP and OSPF to calculate the shortest path. In case of OSPF we are using the metrics that takes the channel throughput into account, as in case of RIP we operate with the hop number. Therefore, the channels (edges in graph related terms) with low throughput may not being in use in case of logical structure formed by OSPF. Let us check this assumption. 


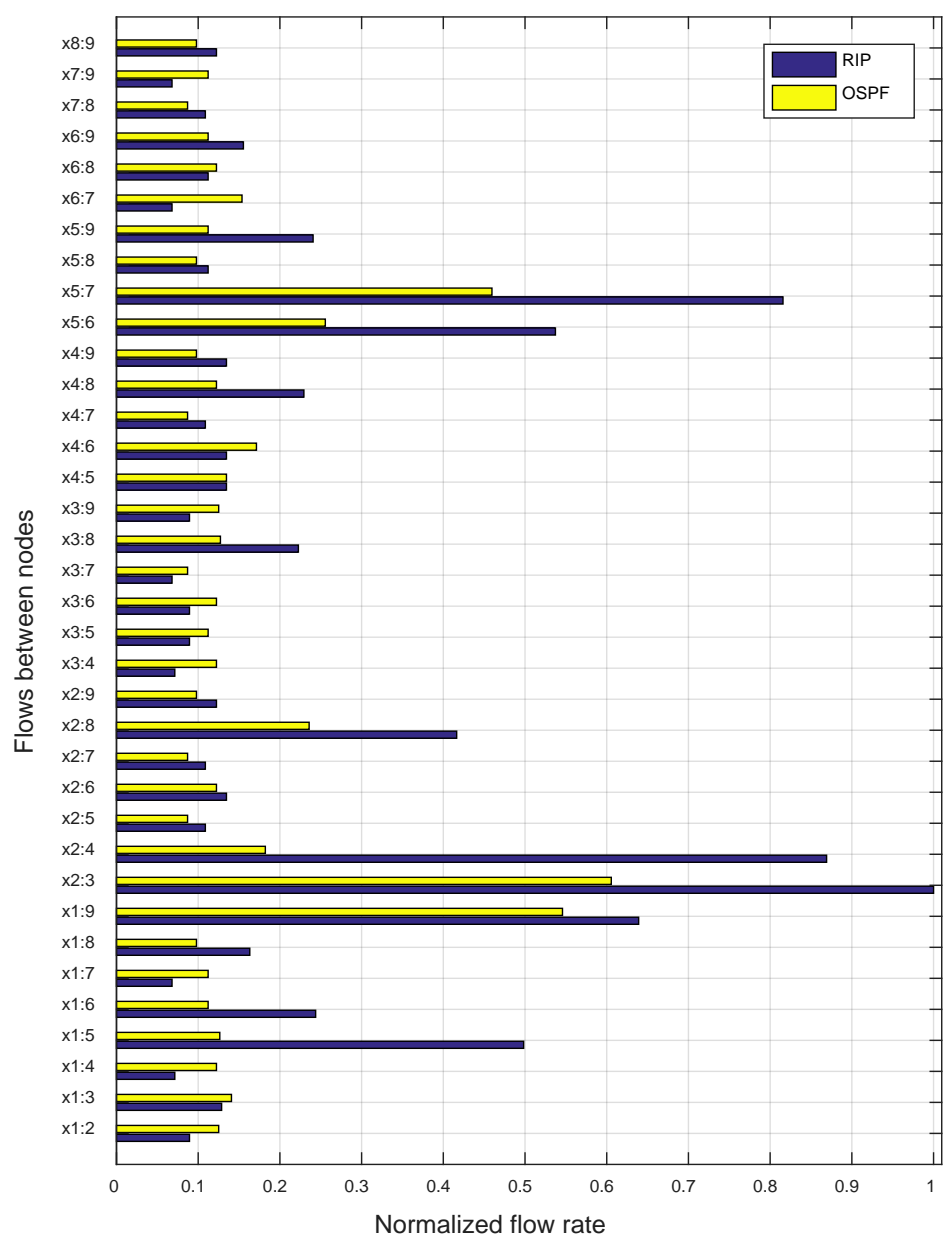

Figure 4: Comparison of the results for RIP and OSPF protocols.

Let us correlate the achieved optimization problem solutions with the network structure in order to analyze the impact of calculated service flows on the use of network resources.

By transmitting flows with calculated rates across the studied network fragment the following resource utilization is observed in each node:

- resources, occupied by outgoing flow;

- resources, occupied by arrival flow;

- resources, occupied by transit flow;

- unused resources.

By transmitting flows across the network with RIP routing the obtained resource allocation has the form shown in Figure 5, a.

Service flows with calculated rates in a logical structure, formed by RIP protocol, use all available network resources (in terms of throughput), because the nodes do not contain unused resources. As shown in Figure 5, node №5 is involved only in transmitting input and output flows, other nodes also transmit transit traffic too. Since each node is the initiator of the flow, the presence of transit flow in the node limits a number of resources that can be given for service flow in this node. We have observed that with increasing of the node (vertex in terms of graph) degree the fraction of transit traffic in this node is also increasing (№1, №4, №6). This happens due to the peculiarities of RIP protocol operation. It calculates routes by the criterion of minimal number of hops, with the maximal utilization of vertices with the highest degree. In such a point, these vertexes (switching centers) can become bottlenecks; therefore, usage of the obtained solution for 
the formulated problem can determine weaknesses in the design of the physical structure of the telecommunication network. It is recommended in physical structures containing vertexes with mixed degrees to identify those for which the degree is maximal and provide the available throughput of adjacent channels slightly higher than the rest. This will enable the reserved throughput for transit traffic and will reduce its impact on the service flow rate, which is formed in this node.

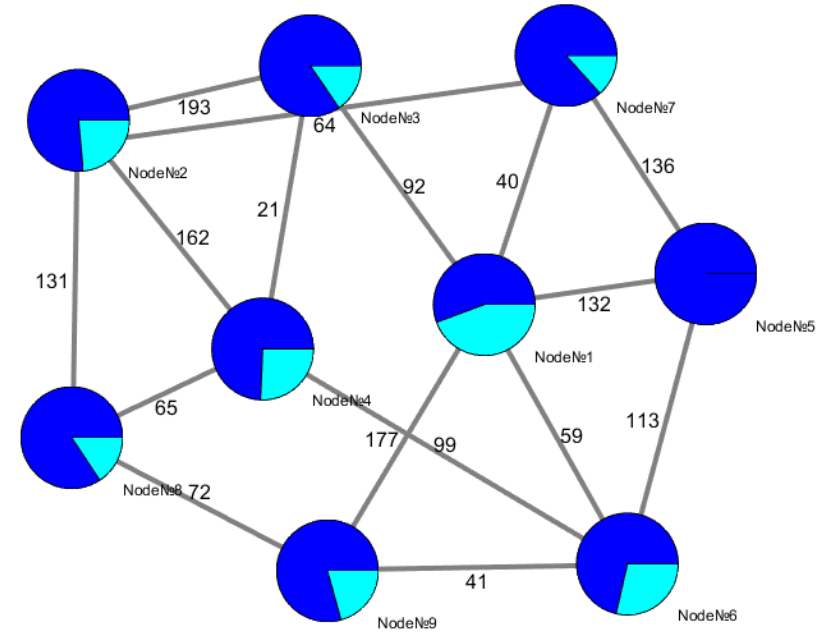

a)

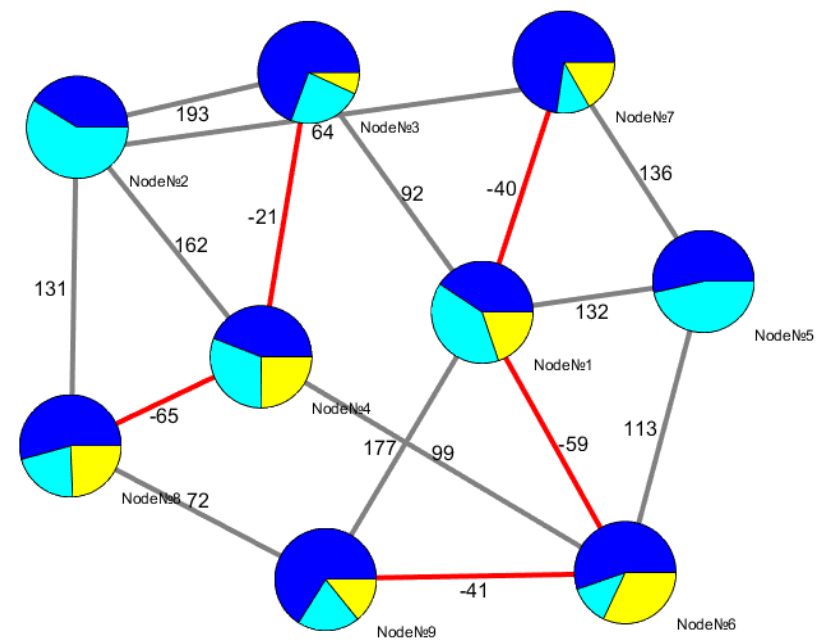

b)

Figure 5: The network resources allocation in a logical structure formed: a) according to the RIP protocol; b) according to the protocol OSPF (blue - service flow, light blue - transit traffic from other service flows)

The formulated problem is also solved in the case of forming the logical structure by OSPF protocol. The obtained results differ from described above for RIP protocol. As it can be seen in Figure 5, b, almost every node contains unused resources.

Detailed analysis showed that unused resources are present at nodes, which adjacent channels have minimal throughputs. They are rejected by OSPF protocol as expected during the shortest paths selection (edges $e_{1,6}, e_{1,7}, e_{3,4}, e_{4,8}, e_{6,9}$, marked with negative weights in Figure 5 , b). The explanation is the protocol OSPF selects routes nonmetering the other flows that are held on separate channels. Also, as it is shown in Figure 6, b a significant fraction of transit passes through the nodes №2 and №5 because their adjacent channels have the highest throughputs. OSPF protocol discovers the redundant edges in the physical structure of the network that can be removed. On the other hand, the result shows the inconsistency of the physical and logical structure. We recommend to use weighted multipath routing for maximal physical resources utilization in that case.

Thus, we observe the logical structure formed by routing protocol imposes significant limitations on the availability of physical resources for service flows transmission.

\section{Conclusions}

This paper proposes a solution to the important problem of resource allocation among network service flows provided them equal competition for the given physical resources in order to estimate the maximum number of clients using the service with multiple levels of service quality. The task is formulated in terms of graph theory and solved by the method of linear programming in the case of the telecommunications network with 9 nodes and 16 channels of predetermined throughput. 
The paper considers the impact of the logical structure on the availability of network resources. Problem solution depicts that in the case of forming the logical structure by RIP protocol service flows in the equal competition reach their maximum rate and use all the available network resources. However, for a logical structure formed by OSPF protocol not all available physical resources are used, as the routing protocol ignores the edges with minimal throughput, and therefore these edges are not included in the problem solution. This fact makes it possible to identify redundancy in the physical topology and reduce the cost of the designed network or change the routing policy by introducing k-path routing through unused channels, thereby increasing its performance.

The further work is related to developing methods of service quality assurance to end users within the calculated rate of the group service flow (each user will obtain the portion on the basis of its priority). It is assumed the use of nonlinear optimization methods to describe the models of service flows distribution among the end users.

\section{Acknowledgements}

This research is supported by the project entitled "Methods for designing the heterogeneous information and communication system to deploy the software defined multi-purpose 5G networks", funded by Ukrainian government.

\section{References}

[1] J. He, J. Rexford, and M. Chiang, "Design principles of manageable networks", Princeton University Computer Science, Technical Report TR-770-06, Oct. 2006.

[2] L. Beril Toktay and R. Uzsoy, "A Capacity Allocation Problem with Integer Side Constraints", European Journal of Operational Research, Volume 109, Issue 1, pp. 170-182, 1998.

[3] L.F. Ochoa, C.J. Dent and G. P. Harrison, "Distribution Network Capacity Assessment: Variable DG and Active Networks", IEEE Transactions on Power Systems, vol. 25, pp. 87-95, 2010.

[4] Sayan Sen Sarma, Goutam Chakraborty, "Queuing model-based optimal traffic flow in a grid network”, IEEE International Conference on Advanced Networks and Telecommunications Systems (ANTS), 2015, pp. 1-3.

[5] A.V. Lemeshko, H.D.K. Al-Janabi and O.M. Khodzhaev, "A Mathematical Model of the Centralized Distribution of the Downlink WiMAX Resources on the Channel and Network Layers of the OSI Model", Telecommunications Problems, No. 1 (18), 1 p. 52-71, $2016 . \quad$ [Online]. Available: http://pt.journal.kh.ua/2016/1/1/161_lemeshko_wimax.pdf.

[6] Qin Ba, Ketan Savla and Giacomo Como, "Distributed optimal equilibrium selection for traffic flow over networks”, 54-th IEEE Conference on Decision and Control (CDC), pp. 6942-6947, 2015.

[7] Bonnans, J. Frédéric, Gilbert, J. Charles, Lemaréchal, Claude, Sagastizábal, Claudia A., “Numerical optimization: Theoretical and practical aspects", Universitext (Second revised ed. of translation of 1997 French ed.), Berlin, Springer-Verlag, ISBN 3-540-35445-X. MR 2265882.

[8] M. Klymash, O. Lavriv, B. Buhyl, Y. Danik, "Service quality oriented method of multiservice telecommunication networks design," in Modern Problems of Radio Engineering Telecommunications and Computer Science (TCSET), 2012 International Conference on, 2012.

[9] P. Huskov, O. Lavriv, and M. Klymash, "The concept of services assurance in heterogeneous service-oriented systems," in 2016 Third International Scientific-Practical Conference Problems of Infocommunications Science and Technology (PIC S\&T), 2016, pp. 24-26.

[10] O. Lavriv, B. Buhyl, P. Huskov, and R. Bak, "Heterogeneous network capacity distribution among service flows," in 2017 14th International Conference The Experience of Designing and Application of CAD Systems in Microelectronics (CADSM), 2017, pp. 173-175.

[11] Bioinformatics Toolbox. Documentation. [Online]. Available: https://www.mathworks.com/ help/ bioinfo/index.html

[12] Optimization Toolbox. Documentation. [Online]. Available: https://www.mathworks.com/help/optim/. 